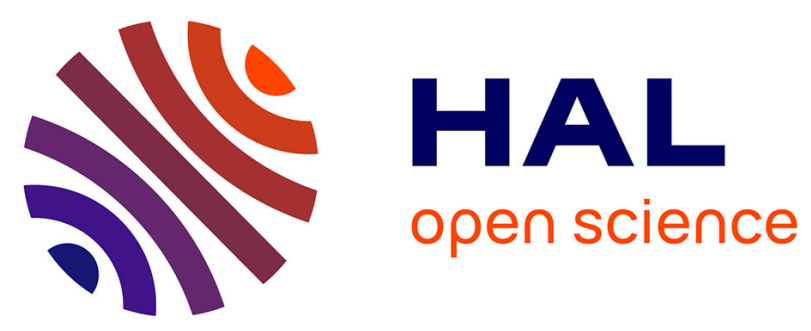

\title{
Control of a Magnetic Microrobot Navigating in Microfluidic Arterial Bifurcations through Pulsatile and Viscous Flow
}

\author{
Karim Belharet, David Folio, Antoine Ferreira
}

\section{To cite this version:}

Karim Belharet, David Folio, Antoine Ferreira. Control of a Magnetic Microrobot Navigating in Microfluidic Arterial Bifurcations through Pulsatile and Viscous Flow. IEEE/RSJ International Conference on Intelligent Robots and Systems (IROS'2012), Oct 2012, Vilamoura, Algarve, Portugal. pp.2559-2564, 10.1109/IROS.2012.6386030 . hal-00714370

\author{
HAL Id: hal-00714370 \\ https://hal.science/hal-00714370
}

Submitted on 8 Jan 2013

HAL is a multi-disciplinary open access archive for the deposit and dissemination of scientific research documents, whether they are published or not. The documents may come from teaching and research institutions in France or abroad, or from public or private research centers.
L'archive ouverte pluridisciplinaire HAL, est destinée au dépôt et à la diffusion de documents scientifiques de niveau recherche, publiés ou non, émanant des établissements d'enseignement et de recherche français ou étrangers, des laboratoires publics ou privés. 


\title{
Control of a Magnetic Microrobot Navigating in Microfluidic Arterial Bifurcations through Pulsatile and Viscous Flow
}

\author{
Karim Belharet, David Folio and Antoine Ferreira
}

\begin{abstract}
Navigating in bodily fluids to perform targeted diagnosis and therapy has recently raised the problem of robust control of magnetic microrobots under real endovascular conditions. Various control approaches have been proposed in the literature but few of them have been experimentally validated. In this paper, we point out the problem of navigation controllability of magnetic microrobots in high viscous fluids and under pulsatile flow for endovascular applications. We consider the experimental navigation along a desired trajectory, in a simplified millimeter-sized arterial bifurcation, operating in fluids at the low-Reynolds-number regime where viscous drag significantly dominates over inertia. Different viscosity environments are tested under a systolic pulsatile flow compatible with heart beating. The control performances in terms tracking, robustness and stability are then experimentally demonstrated.
\end{abstract}

\section{INTRODUCTION}

Recent developments of microelectromechanical systems (MEMS) make possible to fabricate untethered biomedical microrobots that can be injected intravenously to accomplish targeted drug delivery tasks. Benefiting from their small size and biocompatible properties, swimming microrobots are able to reach and function within regions that are unsuitable for traditional devices, which make them a good choice for controlled drug delivery microrobot. Most of swimming approaches consequently rely upon magnetic fields to wirelessly transmit power to the microrobot. This proof-of-concept was first studied using electromagnets [1] and superconducting magnets [2], [3] in phantom devices. Rapidly, magnetic manipulation of therapeutic ferromagnetic nanoparticles (magnetic drug delivery) has progressed from animal to human clinical trials for shallow targets. It is currently limited to static magnets as yet there is no active feedback control in this arena. Recently, magnetic microrobots have received a lot of attention since they are able to provide large motion forces and move in liquid environments with very low (less than one) Reynolds number environment (i.e., the ratio of inertial force to viscous force). Magnetic propulsion and steering for ferromagnetic microparticles, also has been employed [4] in which the magnetic force and torque of a microrobot were induced independently by Maxwell and Helmhotz coil fields [5], [6]. Magnetic helical medical microrobots, inspired by the propulsion of bacterial flagella, are promising for use in open fluids for destroying kidney stones in real human body [4], or for surgery in ophtalmic procedures [7], [8]. Finally, magnetotactic bacteria

The authors are with the Laboratoire PRISME, Ecole Nationale Supérieure d'Ingénieurs de Bourges, 88 Bld Lahitolle, F-18020 Bourges; France. E-mail: $\{$ first Name. Name\}@ensi-bourges.fr actuated thanks to embedded or attached ferromagnetic material has been demonstrated [9]. All these contributions point out the problem of navigation controllability of magnetic microrobots in high viscous fluids and under pulsatile flow when experimental endovascular applications are considered. Recently, a new approach referred to as magnetic resonance navigation has been proposed to steer and track in real time endovascular magnetic carriers in deep tissues to target areas of interest [10]. As it focuses on in-vivo feasibility studies of the microrobot pulling concept, developed model is linear [11] and in turn the synthesis of control laws relies on linear PID approaches. Authors in [12] report instabilities and important oscillations around the equilibrium, especially when the blood stream is modeled as a pulsatile flow. The experiments figure out a lack of robustness to noise and unmodeled dynamics. To overcome these limitations, we analyze in this study a magnetic microrobot body navigating within a microfluidic chip under real physiological conditions. We consider the experimental navigation along a desired trajectory, in a simplified arterial bifurcation geometry, operating in fluids at the low-Reynolds-number regime where viscous drag significantly dominates over inertia. Different viscosity environments are tested (ranging from 100\% water-to- $100 \%$ glycerol) under a systolic pulsatile flow. We demonstrate experimentally that the generalized predictive controller (GPC), developed previously in [13], is sufficiently robust against nonlinear model uncertainties (e.g. drag force and viscosity), external perturbations (systolic pulsatile flow) and noisy trajectory tracking measurements.

This paper is organized as follows. First, Section II introduces an experimental setup that is used to operate magnetic microrobots in microfluidic arterial bifurcations. Section 3 provides an overview of the physics of control of a microrobot using generalized predictive control (GPC) strategy. Section 4 presents experiments to illustrate the efficiency and robustness of the predictive navigation control approach w.r.t. different viscous medium and pulsatile flows. Finally, Section 5 discusses the advantages of the predictive navigation and some limitations related to the complexity of endovascular structure network. This paper is concluded in Section 6.

\section{EXPERIMENTAL SETUP}

\section{A. Magnetic Setup}

The experimental setup used to generate the 3D controlled magnetic fields is shown in Fig. 1(a) and has been developed specifically for the experiments (AeonScientific ${ }^{T M}$, ETH Zurich) [14]. The system consists of three nested sets of Maxwell coils and one nested set of Helmholtz 


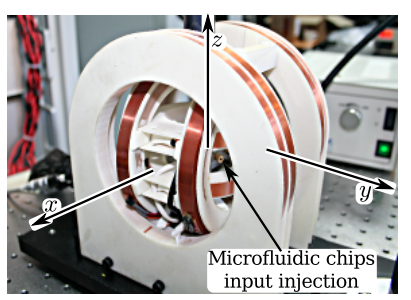

(a)

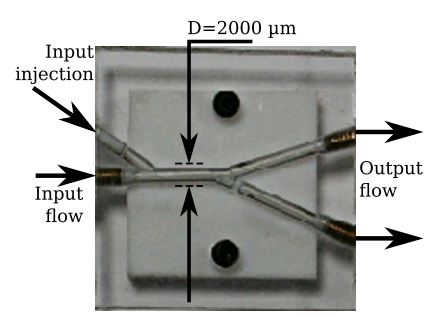

(b)
Fig. 1. (a) 3D Maxwell-Helmholtz coils setup and (b) Y-shaped microfluidic arterial bifurcation chip (diameter: $2 \mathrm{~mm}$ ).

coils. Usually, Helmholtz and Maxwell pairs are combined coaxially such that the magnetic field and magnetic gradient field can be controlled independently in the center of the workspace [7], [15]. Such arrangement allows to generate a uniform magnetic gradient field pointing in $x-, y-$, and $z$-axis direction. Each set of Maxwell coils generates a magnetic field that is optimally uniform in the center of workspace, aligned with the axis of the coils, and varying linearly with the electrical current flowing through the wire. The three sets are arranged orthogonally such that the magnetic gradients vector can be aligned arbitrarily, with each Maxwell pair corresponding to one basis direction of the field vector. Magnetic gradient forces will thus be exerted on the magnetic microrobot that is inside the Y-channel of the microfluidic chip, as depicted in Fig. 1(b). Homogeneous magnetic flux densities and gradients of up to $300 \mathrm{mT}$ and $350 \mathrm{~m} / \mathrm{T}$, respectively, can be generated in a workspace of $20 \mathrm{~mm} \times 20 \mathrm{~mm}$. The Helmholtz coils corresponds to the $x$-axis in order to magnetize ferromagnetic microrobots with low magnetization values. However, as the direction of the magnetic field and the magnetic gradient are dependent on each other, it means that the system is non-holonomic meaning that a non-spherical object cannot be steered in a controllable way. That is why we choose a spherical neodymium-iron-boron (neodymium magnet) as microrobot body (termed microrobot throughout the text). Each set of Helmholtz and Maxwell pairs are driven by PWM analog servodrives (Aeon Scientific) capable of 12 continuous current controlled by a computer through the Labview software interface. The system is set up on an CCD high-resolution miniature microscope camera (TIMM 400) providing up to $26 \mathrm{~mm} \times 20 \mathrm{~mm}$ field of view. The motion of magnetic microrobot is measured by real-time processing the video images acquired by the microscope camera using Labview computer program with a submicrometer resolution. A robust tracking algorithm has been developed.

\section{B. Vascular bifurcation-like environment condition}

To mimic the endovascular navigation environment, we developed a microfluidic chip with a small vascular bifurcation configuration to reproduce real parent-daughter branching vessels as illustrated in Fig. 1(b). The liquid is pumped using a pulsatile blood pump (Harvard Apparatus) that mimics the blood flow in the human cardiovasculature system (non-

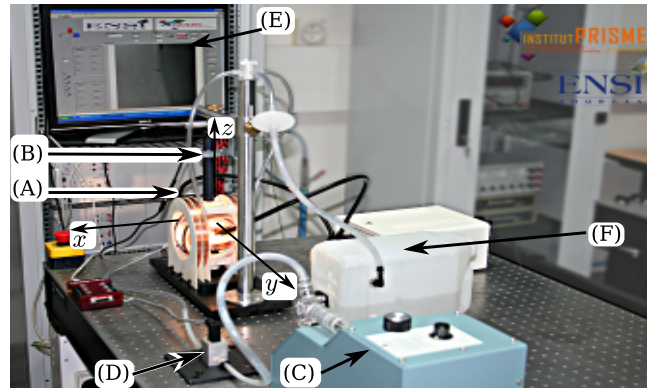

(a)

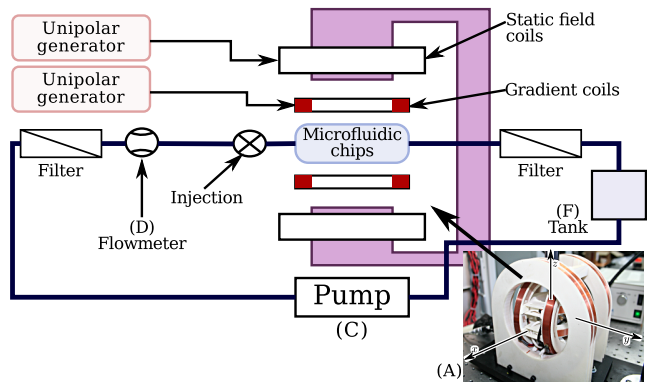

(b)

Fig. 2. Overall experimental system setup: (A) Pair of coils; (B) Miniature Microscope; (C) Havard pump; (D) flowmeter; (E) LabView PC software; and $(\mathrm{F})$ liquid tank;

Newtonian fluids such as blood and pulsatile flows), as shown in Fig. 2. Such pulsatile pump is able to generate a sinusoidal flow with negative values to imitate the arterial reflux. To prevent their suction by the pump, the microrobot is blocked in the microfluidic chip using two PVDF security microfilters. A bi-directional flowmeter is placed at the input of the microfluidic chip. Finally, different water/glycerol liquids mixtures are tested (viscosity variation) to simulate different human blood viscosities. A plastic tank is used to store the various mixtures of water and glycerol pumped, and also to retrieve the liquid leaving the vascular phantom. Table I summarizes the different experimental conditions.

TABLE I

EXPERIMENTAL CONDITIONS $\left(\mathrm{at}^{\dagger} T=20^{\circ} \mathrm{C}\right)$.

\begin{tabular}{|ll||l||l|}
\hline $\begin{array}{l}\text { Microsphere } \\
\text { neodymium magnet } \\
\text { (NdFeB }-35)\end{array}$ & radius & $r$ & $250 \mu \mathrm{m}$ \\
\cline { 2 - 4 } & density & $\rho_{m}$ & $7500 \mathrm{~kg} / \mathrm{m}^{3}$ \\
\hline Microfluidic & radius & $R$ & $1000 \mu \mathrm{m}$ \\
\hline \hline Water & density & $\rho_{\mathrm{f}, \mathrm{w}}$ & $1000 \mathrm{~kg} / \mathrm{m}^{3}$ \\
\cline { 2 - 4 } & viscosity & $\eta_{\mathrm{w}}$ & $10.05 \cdot 10^{-4} \mathrm{~Pa} \cdot \mathrm{s}$ \\
\hline \hline $\begin{array}{l}\text { Glycerol } \\
\text { (glycerin) }\end{array}$ & density & $\rho_{\mathrm{f}, \mathrm{g}}$ & $1260 \mathrm{~kg} / \mathrm{m}^{3}$ \\
\hline
\end{tabular}

${ }^{\dagger}$ The experiments are conducted at air conditioned.

\section{MAGNETIC MICROROBOT NAVIGATING IN VISCOUS MICROFLUIDIC ENVIRONMENT}

\section{A. Modeling}

The microrobot body is modeled by a magnetic microsphere with a high saturation magnetization value as depicted on Fig. 3. Actuated by external magnetic gradients, the microrobot will potentially experience magnetic, 


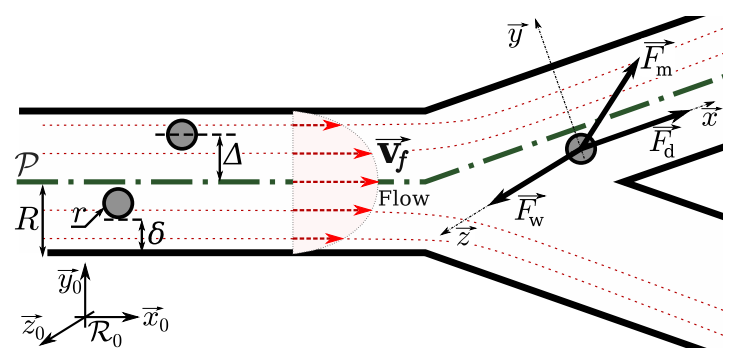

Fig. 3. Microrobot navigating in a microfluidic arterial bifurcation.

gravitational, contact, electrostatic, van der Waals and drag microforces that affect the microrobot's motion. The effects of these forces are explained in detail in [16].

As model simplification, we notice that close to the centerline path $\mathcal{P}$ of the vessel $(\Delta=0)$, the electrostatic $\left(\mathbf{F}_{\mathrm{el}}\right)$ and the van der Waals $\left(\mathbf{F}_{\mathrm{vdw}}\right)$ microforces are negligible compared to the other relevant forces [16]. Furthermore, we assume that the microrobot is never in contact with the microfluidic's vessel wall as its position tracks the centerline of the vessel. Finally, the apparent weight force $\left(\mathbf{W}_{a}\right)$ of the microrobot is counterbalanced by the sustentation magnetic force in $z$-axis. Due to the size of the microsphere we neglect the effect of Brownian motion. Finally, a simplified sphere model can be adopted:

$$
m \frac{d \mathbf{v}}{d t} \cong \mathbf{F}_{\mathrm{m}}+\mathbf{F}_{\mathrm{d}}
$$

The magnetic gradients generated by the coils induces a motive force:

$$
\mathbf{F}_{\mathrm{m}}=V_{\mathrm{m}}(\mathbf{M} . \nabla) \mathbf{B},
$$

where $V_{\mathrm{m}}$ is the magnetic volume of the magnetic material, $\mathbf{M}$ its magnetization, $\mathbf{u}=\nabla \mathbf{B}$ is the controlled magnetic gradient. If the microrobot moves slowly (i.e. with a Reynolds number $\mathrm{Re} \ll 1$ ) the hydrodynamic drag force can then given by Stokes' law:

$$
\mathbf{F}_{\mathrm{d}} \approx-6 \pi \eta_{\mathrm{f}} r\left(\mathbf{v}-\mathbf{v}_{\mathrm{f}}\right) .
$$

where $\eta_{\mathrm{f}}$ is the flow viscosity, $r$ the radius of the microsphere, $\mathbf{v}$ is the velocity of the microrobot, and $\mathbf{v}_{\mathrm{f}}$ the fluid flow. The pulsative (blood) flow's velocity $\mathbf{v}_{\mathrm{f}}$ is usually approximated by an affine combination of a time-varying periodic flow with a spatial parabolic profile.

Finally, it can been shown that a state-space representation could be deduced from the equation (1) and is expressed as follows [16]:

$$
(S)\left\{\begin{aligned}
\dot{\mathbf{x}} & =\mathbf{v} \\
\dot{\mathbf{v}} & =f(\mathbf{x}, \mathbf{v})+\alpha \mathbf{u} \\
\mathbf{y} & =\mathbf{x}
\end{aligned}\right.
$$

where the control input $\mathbf{u}=\nabla \mathbf{B}$ is the magnetic gradient, $\alpha$ is a parameter obtained from (2), and $f(\mathbf{x}, \mathbf{v})$ is function that stems from the projection of external forces $\mathbf{F}_{\text {Ext. }}$. In this paper we aim to embed the system model (4) in a predictive controller to follow efficiently a pre-planed path $\mathcal{P}$ extracted with the method proposed in [13].

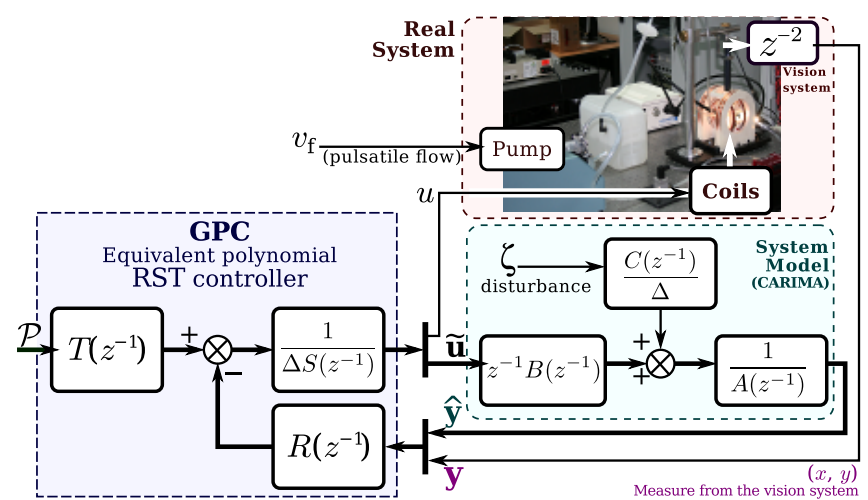

Fig. 4. Predictive navigation control (GPC scheme).

\section{B. Navigation Control}

To ensure a robust and smooth conveyance of the microrobot to its destination it is necessary to drive its pose $\mathbf{x}$ between a planned pathway $\mathcal{P}$ and the observation through a navigation control module. To our knowledge, most of feedback controller schemes designed in the scope of microrobotics facing drag are simple PID approaches [11]. In [16], the authors proposed to design a controller based on the backstepping approach that ensures Lyapunov stability. However, such control scheme could be regarded as low level controller, that is guaranteeing local stability. It is then difficult to overcome the relevant system constraint using only such robust control strategies. Therefore, to design our navigation control module we have considered predictive control strategies. Predictive control has become a significant research interest powered by a stream of successful industrial applications [17]. The key idea of predictive control schemes are to predict the behavior of the system over a given time horizon. From this prediction, a control is computed by minimizing a quadratic cost function. In particular, when focusing on linear discrete time transfer function models and quadratic cost functions, one of the best known approaches is the Generalized Predictive Control (GPC) introduced by Clarck et al. [18]. In GPC scheme design the system is usually modeled using the model Controlled Auto-Regressive Integrated Moving-Average (CARIMA), as illustrated in Fig. 4. This CARIMA system model is obtained from the state-space representation defined in (4) [19]. The proposed GPC is then obtained by minimizing the following criterion:

$$
J_{\{N, \lambda\}}=\sum_{j=0}^{N}\left(\widehat{y}_{(t+j)}-\mathcal{P}_{(t+j)}\right)^{2}+\lambda \delta \mathbf{u}^{2}(t)
$$

where $\widehat{y}_{(t+j)}$ is the optimum predicted output of the system at time $t+j, \mathcal{P}_{(t+j)}$ is the future reference, $N>0$ define the size of the prediction horizon; and $\lambda>0$ is the control weighting. Hence, a RST polynomial structure is added to determine a relation between the output $\mathbf{y}$, the control signal $\mathbf{u}$ and the reference $\mathcal{P}$ (see Fig. 4). 


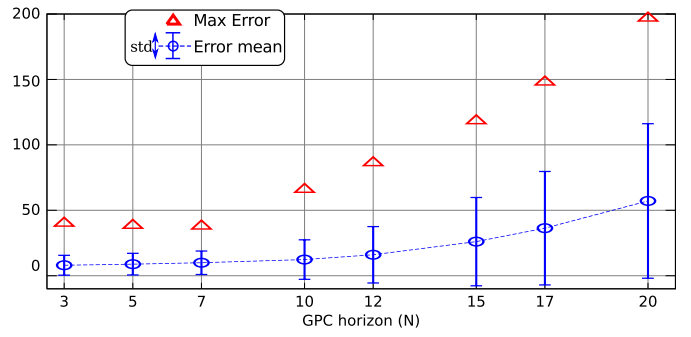

(a) The circle/bar is the mean/std of $\Delta ; \Delta$ is the maximum of $\Delta$.

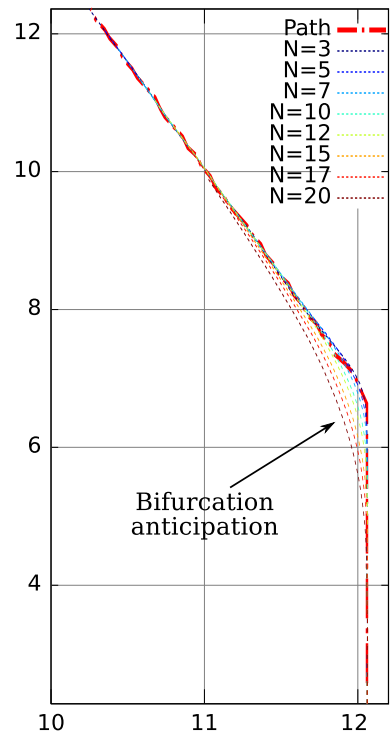

(b)

Fig. 5. Simulation results with pulsatile flow: (a) Path tracking error $\Delta$ (in $\mu \mathrm{m}$ ) w.r.t. GPC horizon $N$; and (b) microrobot trajectory showing anticipation behavior

\section{Simulations}

The proposed GPC navigation strategy has been first validated in simulation in presence of a systolic pulsatile flow. The pulsative blood's velocity is modeled by an affine combination of a time-varying periodic flow with a spatial parabolic shape. So as to simplify the analytical expression, but with no loss of generality, we only consider the first terms in the time-varying Fourier series of the physiological pulse. In the case of an artery, such an approximation leads to:

$$
v_{f}(t)=V_{f}\left(1+a_{f} \sin \left(w_{f} t+\phi_{f}\right)\right)\left[1-\left(\frac{R-\Delta-r}{R}\right)^{2}\right]
$$

Fig.5 shows the impact of the GPC horizon $N$ on the path tracking error. Note that, as the defined reference path $\mathcal{P}$ is centered in the vessel, this path tracking error is defined as: $\Delta_{k}=\left\|\mathbf{y}_{k}-\mathcal{P}_{k}\right\|$, with $\mathbf{y}_{k}=\left(x_{k}, y_{k}\right)^{T}$ is the measured microrobot position at time $t_{k}$, and $\mathcal{P}_{k}=\left(x_{k}^{\star}, y_{k}^{\star}\right)^{T}$ is the corresponding point on the centerline path $\mathcal{P}$. We can notice that for $N>10$, the error $\Delta$ increase gradually until to be in the close vicinity of the vessel wall. This behavior illustrates the nature of anticipation of the GPC strategy. Increasing $N$ at high values leads to overanticipate the path behavior that results in poor tracking performances, and potentially, contact effects with vessel walls(Fig.5(b)).

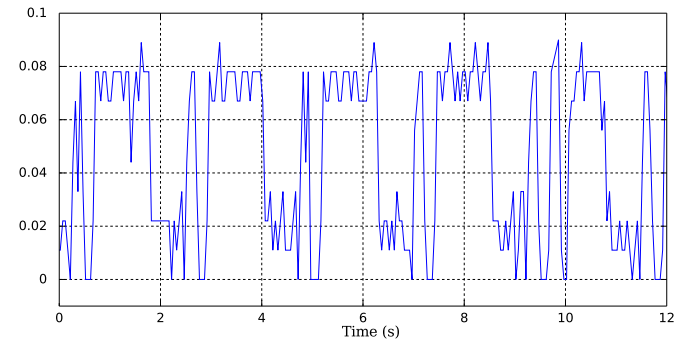

Fig. 6. Experimental pulsatile flow rate generated by the blood pump. $\left(Q_{\mathrm{f}, \max }=0.167 \mathrm{~L} / \mathrm{s}, Q_{\mathrm{f}, \text { mean }}=0.0465 \mathrm{~L} / \mathrm{s}, Q_{\mathrm{f}, \mathrm{RMS}}=0.0563 \mathrm{~L} / \mathrm{s}\right)$

\section{EXPERIMENTAL RESULTS}

Fig. 2 shows the overall experimental setup used for the microrobot control in a microchannel $(R=1000 \mu \mathrm{m})$ environment using magnetic gradients. In these experiments, a spherical $(r=250 \mu \mathrm{m})$ neodymium magnet was used as microrobot body. As previously presented, an endovascular system is reproduced using an Y-shaped vascular phantom to mimic a branching vessel. Several experiments were conducted with different mixtures of water and glycerol (see table II) to test our proposed predictive control strategy in different viscous mixtures.

TABLE II

AqueOus GLYCEROL SOLUTIONS (at $T=20^{\circ} \mathrm{C}$ ).

\begin{tabular}{|l|c|c|c|}
\hline Glycerol (\% weight) & $50 \%{ }^{\ddagger}$ & $80 \%$ & $100 \%$ \\
\hline Density $\left[\mathrm{kg} / \mathrm{m}^{3}\right.$ ] & 1130 & 1208 & 1260 \\
\hline Viscosity $[\mathrm{Pa} \cdot \mathrm{s}]$ & $60 \cdot 10^{-4}$ & 0.06 & 1.41 \\
\hline
\end{tabular}

\section{A. Experimentation protocol}

First, the centerline reference path $\mathcal{P}$ is computed automatically using the algorithm developed in [13]. Then, the pulsatile blood pump is started to fill out the pipes and the vascular phantom with different mixture of water/glycerol solutions. Especially, the volumetric flow rate is settled at $Q_{\text {Human }} \approx 0.06 \mathrm{~L} / \mathrm{s}$ to simulate the human cardiovascular pulsatile flow. After these preliminary steps, the microsphere is injected into the vascular phantom (in the region of interest) using a syringe. Finally, the GPC navigation scheme is initialized via the LabView control interface.

\section{B. Static flow experiments}

The first experiments are conducted within a static flow to tune the set of parameters of the predictive controller. Fig .7(a) shows the impact of the prediction horizon $N$ on the path tracking error for different aqueous glycerol mixtures (see Tab. II). It should be noticed that the overall path tracking error is satisfactory as the average position error remains close to the centerline $\mathcal{P}$. As previously stated in simulation in section III-C, the prediction horizons $N>10$ once again do not provide significant contribution, whereas increasing the prediction horizons increase the computational time. One can see that the distance to the pipe wall $\delta$ (see also Fig. 3) is globally about $400 \mu \mathrm{m}$ ensuring that the microrobot 


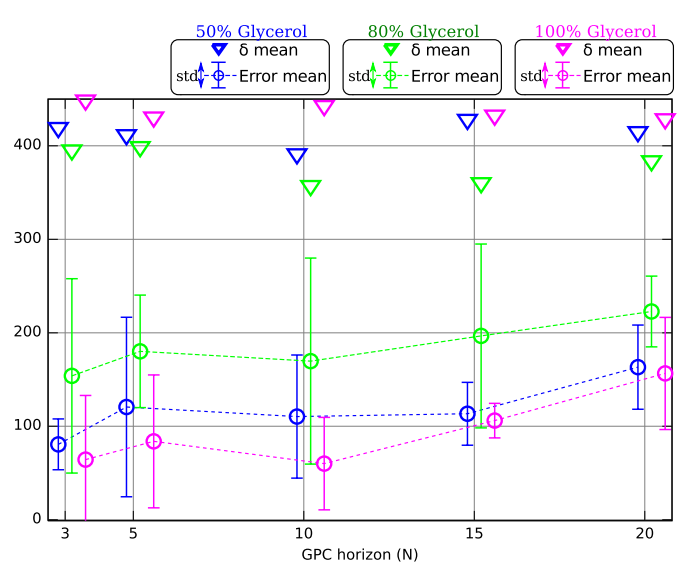

(a)

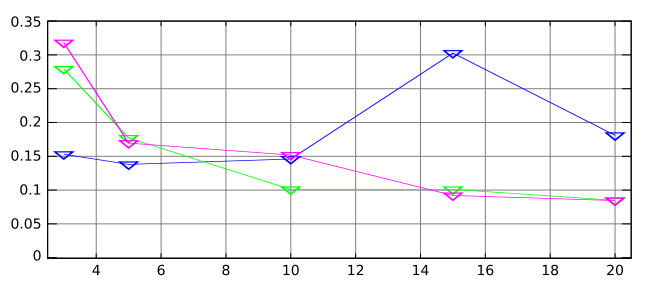

(b)

Fig. 7. Path tracking w.r.t. GPC horizon $N$, with water and $50 \%, 80 \%$ and $100 \%$ glycerol mixtures, within static flow. (a) Path tracking error and mean of the distance to vessel wall $\delta$; (b) maximum generated $\nabla \mathbf{B}$.
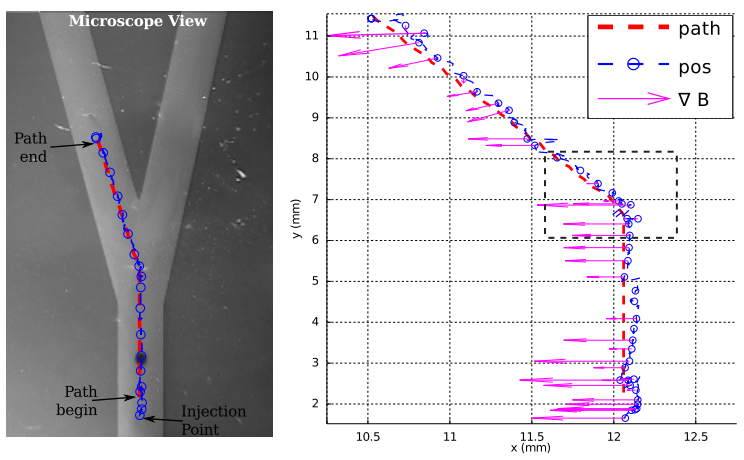

Fig. 8. Path tracking with GPC horizon $N=10$, and blood flow condition, within static flow.

is never in contact with the vessel wall. Fig. 8 illustrates the case where the GPC prediction horizon is set to $N=10$, and using a $50 \%$ water $-50 \%$ glycerol mixture, that is close to blood flow condition.

\section{Pulsatile flow experiments}

Experiments are also conducted considering pulsatile flow to validate the proposed predictive navigation control strategy. Fig.6 illustrates a sample record of such pulsatile flow. Fig. 9 depicts the overall tracking error $\Delta$ for different the GPC horizon values $N$ within an aqueous solution of $50 \%$ and $80 \%$ of glycerol. As previously stated, increasing the prediction horizon increases the anticipatory behavior of the predictive navigation scheme. Comparing to the static flow experiments, one can see that the presence of the pulsatile flow, the amplitude value of the applied gradients increases

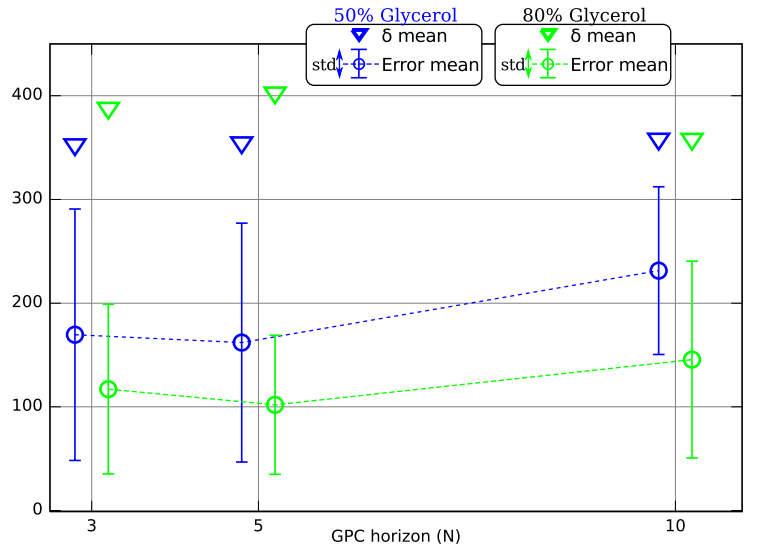

(a)

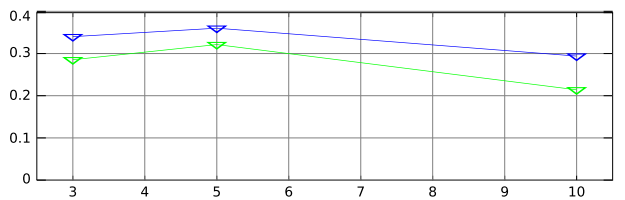

(b)

Fig. 9. Path tracking w.r.t. GPC horizon $N$, with water and $50 \%$ and $80 \%$ glycerol mixtures, within pulsatile flow. (a) Path tracking error and mean of the distance to vessel wall $\delta$; and (b) maximum generated $\nabla \mathbf{B}$.
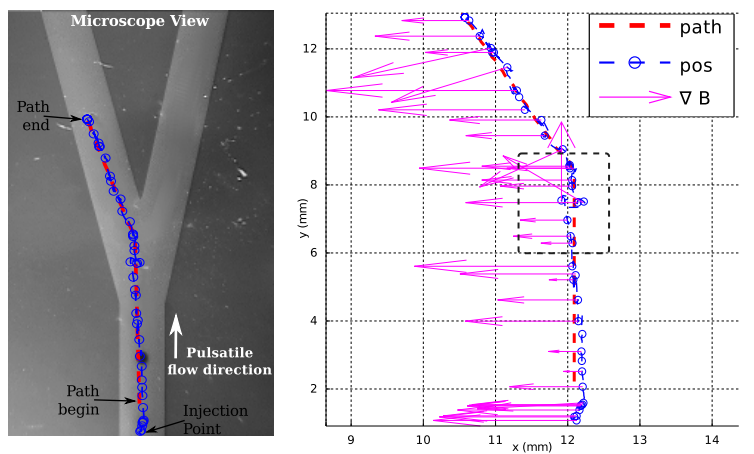

Fig. 10. Path tracking with GPC horizon $N=10$, and blood flow condition, with pulsatile flow.

greatly Fig. 9(b)). It can be explained by the need to counterbalance the antagonistic pulsative flow during navigation. Fig. 10 shows the case where the $N=10$ using a $50 \%$ water $-50 \%$ glycerol mixture. Once again, the experimental results illustrate that the controller remains robust even in the presence of pulsatile flow for different mixtures.

\section{DISCUSSION}

The experiments demonstrate that the maximum computed magnetic gradients are below $\|\nabla \mathbf{B}\|<350 \mathrm{mT}$ (see Fig. 7(b) and Fig. 9(b)) leading to magnetic force values (2) $\left\|\mathbf{F}_{\mathrm{m}}\right\|<30 \mu \mathrm{N}$. The analysis of the results point out several remarks:

-First, as the microrobot navigates through the viscous flow, the flow exerts a drag force that is in the range of $100 \mu \mathrm{N}$ (high viscosity: $100 \%$ glycerol) to $5 \mu \mathrm{N}$ (low viscosity: $50 \%$ glycerol). Such important drag force smooth greatly the motions of the microsphere improving by this way the 


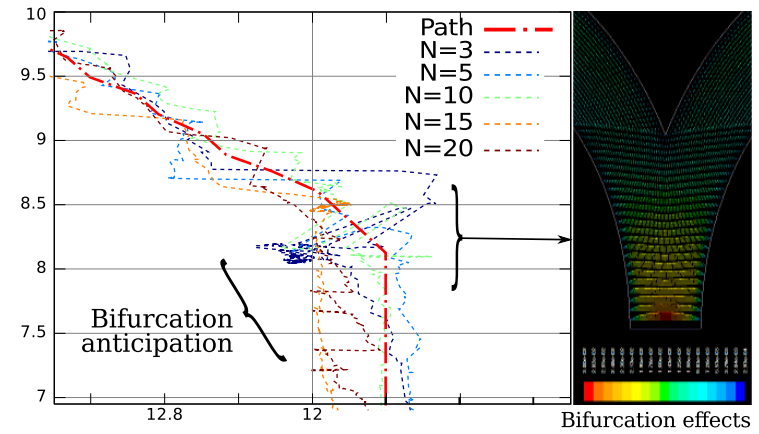

Fig. 11. Zoom on the Y-shape channel bifurcation with $50 \%$ water- $50 \%$ glycerol mixture and varying GPC horizon $N$.

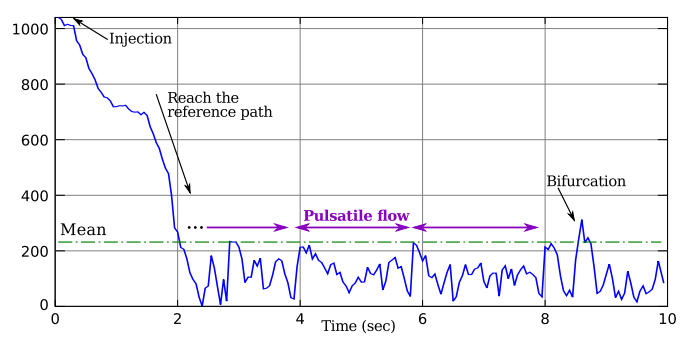

Fig. 12. Tracking error (in $\mu \mathrm{m}$ ) Path tracking with GPC horizon $N=10$, and $50 \%$ water- $50 \%$ glycerol mixture

controllability of the tracking system. Scaling down the size of the microrobot leads to an increase of the magnetic gradient forces due to important hydrodynamic perturbations $\left(\mathbf{F}_{\mathrm{m}}\right.$ evolves in $\sim L^{3}$ while $F_{d} \sim L^{2}$ ).

-Second, the proposed predictive navigation strategy considers linear assumptions on the Stoke's drag force, neglects the electrostatic force, assumes an Newtonian flow throughout the channel (3). The GPC controller proved to be sufficiently robust against modeling uncertainties such as those at the bifurcation area where non-Newtonian model applies (see Fig. 11)and at the injection point that produces flow turbulences (see Fig. 12). In both cases, the microrobot follows the trajectory in a robust way.

-The main drawback of the predictive navigation approach remains on its anticipatory behavior observed when the time horizon $N$ increases. For high prediction horizon values, the microrobot tends to leave the reference path (increasing the tracking error $\Delta$ ) by anticipating the bifurcation branch. For low prediction horizon values, the bifurcation effects [16] influence the microsphere behavior as illustrated in Fig .11.

\section{CONCLUSION}

In this paper, a predictive navigation control of a magnetic microrobot navigating in microfluidic arteria has been developed at microscale, where centerline navigation path extraction and predictive controller have been designed. Several experiments have been conducted with different viscous condition and prediction horizon. Especially, the experimentation results illustrate the efficiency of the proposed GPC scheme, even in presence of pulsatile flow. Future extends will consider other experiments with varying microball and microchannel sizes.

\section{ACKNOWLEDGMENT}

The PhD student K. Belharet is supported by the NanoIRM project, founded by Region Centre and City of Bourges.

\section{REFERENCES}

[1] T. Honda, K. Arai, K. Ishiyama et al., "Micro swimming mechanisms propelled by external magnetic fields," IEEE Trans. Magn., vol. 32, no. 5, pp. 5085-5087, 1996.

[2] E. G. Quate, K. G. Wika, M. A. Lawson, G. T. Gillies, R. C. Ritter, M. S. Grady, and M. A. H. and, "Goniometric motion controller for the superconducting coil in amagnetic stereoaxis system," IEEE Trans. Biomed. Eng., vol. 38, no. 9, pp. 899-905, Sep. 1991.

[3] S. ichi Takeda, F. Mishima, S. Fujimoto, Y. Izumi, and S. Nishijima, "Development of magnetically targeted drug delivery system using superconducting magnet," Journal of Magnetism and Magnetic Materials, vol. 311, no. 1, pp. 367-371, 2007.

[4] L. Edd, S. Payen, B. Rubinsky, M. L. Stoller, and M. Sitti, "Biomimetic propulsion for a swimming surgical microrobot," IEEE/RSJ Int. Conf. on Intel. Robots and Systems, pp. 2583-2588, 2003.

[5] J. J. Abbott, K. E. Peyer, M. C. Lagomarsino, L. Zhang, L. X. Dong, I. K. Kaliakatsos, and B. J. Nelso, "How should microrobots swim?" Int. J. of Robot. Res., Jul. 2009.

[6] M. S. S. Floyd, C. Pawashe, "Two-dimensional contact and noncontact micromanipulation in liquid using untethered mobile magnetic microrobot," IEEE Trans. Robot., vol. 25, no. 6, pp. 303-308, 2009.

[7] K. Yesin, K. Vollmers, and B. Nelson, "Modeling and control of untethered biomicrorobots in a fluidic environment using electromagnetic fields," Int. J. of Robot. Res., vol. 25, no. 5-6, pp. 527-536, 2006.

[8] L. Zhang, J. J. Abbott, L. Dong, B. E. Kratochvil, D. Bell, and B. J. Nelson, "Artificial bacterial flagella: Fabrication and magnetic control," Applied Physics Letters, vol. 94, no. 6, p. 064107, 2009.

[9] S. Martel, M. Mohammadi, O. Felfoul, Z. Lu, and P. Pouponneau, "Flagellated magnetotactic bacteria as controlled MRItrackable propulsion and steering systems for medical nanorobots operating in the human microvasculature," Int. J. of Robot. Res., vol. 28, no. 4, pp. 571-582, 2009.

[10] J. Mathieu, G. Beaudoin, and S. Martel, "Method of propulsion of a ferromagnetic core in the cardiovascular system through magnetic gradients generated by an MRI system," IEEE Trans. Biomed. Eng., vol. 53, no. 2, pp. 292-299, 2006.

[11] S. Tamaz, A. Chanu, J.-B. Mathieu, R. Gourdeau, and S. Martel, "Real-time mri-based control of a ferromagnetic core for endovascular navigation," IEEE Trans. Bio-Med. Eng., vol. 55, no. 7, pp. 1854-1863, July 2008.

[12] H. Choi, J. Choi, K. Cha, L. Quin, J. Li, J. O. Park, S. Park, and B. Kim, "Position stabilization of microrobot using pressure signal in pulsating flow of blood vessel," IEEE Sensors 2010 conference, vol. 18, pp. 723-726, 2010.

[13] K. Belharet, D. Folio, and A. Ferreira, "Three-dimensional controlled motion of a microrobot using magnetic gradients," Advanced Robotics, vol. 25, no. 8, pp. 1069-1083(15), 2011.

[14] M. Kummer, J. Abbott, B. Kratochvil, R. Borer, A. Sengul, and B. Nelson, "OctoMag: An Electromagnetic System for 5-DOF Wireless Micromanipulation,” IEEE Trans. Robot., vol. 26, no. 6, pp. 10061017, Dec. 2010.

[15] J. Abbott, Z. Nagy, F. Beyeler, and B. Nelson, "Robotics in the Small," IEEE Robot. Automat. Mag., p. 92, 2007.

[16] L. Arcèse, M. Fruchard, and A. Ferreira, "Endovascular MagneticallyGuided Robots: Navigation Modeling and Optimization," IEEE Trans. Biomed. Eng., vol. 99, pp. 1-12, 2012, to appear.

[17] S. Qin and T. Badgwell, "A survey of industrial model predictive control technology," Control engineering practice, vol. 11, no. 7, pp. 733-764, 2003.

[18] D. Clarke, C. Mohtadi, and P. Tuffs, "Generalized predictive control - Part I \& II,” Automatica, vol. 23, pp. 137-160, 1987.

[19] K. Belharet, D. Folio, and A. Ferreira, "Endovascular navigation of a ferromagnetic microrobot using MRI-based predictive control," in IEEE/RSJ Int. Conf. on Intel. Robots and Systems, Taipei, Taiwan, Oct. 2010. 